\title{
Towards Successful Social Collaboration in BIM-based Construction: A Review
}

\author{
Che Khairil Izam Che Ibrahim ${ }^{1, *}$, and Sheila Belayutham ${ }^{1}$ \\ ${ }^{1}$ Faculty of Civil Engineering, Universiti Teknologi MARA, 40450 Shah Alam, Selangor, Malaysia
}

\begin{abstract}
The existence of digital applications in construction industry has opened the floodgates to a better integration and collaboration. In particular, Building Information Modelling (BIM) provides construction stakeholders greater visualisation of project information and communications across multiorganisations. Despite the explicit benefits, the study on what it takes to drive the successfulness of social collaboration in BIM still remain elusive. This paper presents a review of the literature to identify the key elements of social collaboration in BIM-based construction projects. The review identified three key elements (i.e. foundational platform, organisational context and behavioural context) which together form the nexus for successful social collaborative practice. The findings in this study extend the collaboration literature in construction domain by providing deeper insights into the characterisation and importance of social collaboration in digital environment.
\end{abstract}

\section{Introduction}

The wider digitalisation across the world is having a direct impact to the construction sector by bringing new technologies. The use of disruptive technology in construction is starting to gain traction, meaning that those responsible for delivering infrastructure have to be more agile and willing to adapt to change in a pragmatic way [1]. The growing pace of technological means role of engineers will change and the blurring of the lines between engineering and technology, can make the opportunities appeal to people with a much wider range of interests to integrate and collaborate.

In particular, Building Information Modelling (BIM) is recognised as one of the initiatives to enhance the platform of collaboration and management of information throughout the building life cycle [2, 3]. Despite its well documented benefits in regard to collaboration, the problem associated with continuous and consistent collaborative practice in BIM project over time still exist $[2,4]$. The possible reasons contributing to this issue are due to the complexity of the organisational behaviour (e.g. lack of collaborative culture and scarcity of knowledge among project teams), self-interest, isolated working practices and trusting the old system to realising the value of digital transformation $[3,5]$. In addition, there are also recognised problems whereby different BIM applications are not communicating with each other due to technical and non-technical factors and hence, limiting the integration process across the engineering disciplines (e.g. civil, electrical, mechanical, automation) $[6,7]$.

Thus, these issues led to the question on what it takes to ensure the successfulness of the collaborative practice within the multi-organizational teams throughout the lifecycle of BIM-based construction project. It is worth highlighting that while collaboration in multi-organisations has proven to be challenging [8], any effort to understand the social-centric collaboration would enhance the understanding of the dynamic collaborative practice of multi-organisations in BIM [3, 9].

Despite prior research on collaboration in construction evidently emphasized the importance of collaborative practice, past efforts have only focused on the element with little empirical evidence and not taking into consideration the social landscape of the digital environment (i.e. BIM). In order to address this gap in the body of knowledge, this study aim to review the key elements of social collaboration in BIM-based construction projects which could form a platform to further understand the movement towards successful social collaboration over the lifecycle of BIM-based projects.

\subsection{The Evolution of the Debate on BIM as a Collaborative Platform}

Research in collaborative practice, particularly in the BIM domain, has gained increasing attention over the past few decades. To trace the patterns and trends, the time can be split into four sub-periods and relevant context of these research can be summarised based on its publication year.

There has been in recent years a greater interest shown by the scholars in the benefits of BIM and collaborative working. The early literature on BIM collaboration is seen to focus on identifying the

\footnotetext{
* Corresponding author: chekhairil449@salam.uitm.edu.my
} 
antecedents and drivers affecting the adoption of collaboration technologies (e.g. BIM). Studies in this period mainly focus on European (e.g. Greece) settings.

In sub period 2011 - 2013, research moves from the understanding of the general concept of BIM collaboration to the investigation of the information exchanges in digital environment, capturing the evidence of BIM collaborative practice as well as focusing on developing a specific framework (e.g. technical BIM requirements and partnering) for BIM implementation. Studies in this period mainly focus on UK, Canada and Australia settings.

In the third sub period (2014-2016), the research has broadened its landscape to more analytical-based studies, testing several concepts and systems for BIM collaboration. Studies begin to use different types of research methods ranging from case studies to modeling. In addition, new lines of research have emerged, where scholars begin to examine the BIM collaborative practice in real cases such as in hospital projects. The literature intensifies its focus on the Nordic countries (e.g. Norway and Finland). Some articles examine the UK and also covering Asia (e.g. Taiwan and Korea) setting for the first time.

In the fourth sub period ( $>$ 2017), the research expands to include the multiple context in understanding the BIM collaborative practice. More studies focusing on the impact of collaborative technologies (i.e. BIM) on the people and organisations begin to develop. A systematic and objective evaluation of available literature on collaboration in construction starts to take place covering relevant research studies in the last 10 years. Articles begin to examine in US and Asia (e.g. China and Hong Kong) settings.

\subsection{Relevant BIM Collaboration in Construction Literature}

There has been much literature about how BIM implementation will influence the success of collaborative practice (e.g. Oraee et al. [9]). However, the intention here is not to review the broader literature on BIM collaboration in construction but to concentrate on past research that focused on the success of collaborative practice in BIM environment.

Particular attention has been given by Singh et al. [10] who developed a theoretical framework of technical requirements for using BIMserver as a multi-disciplinary collaboration platform. They concluded that greater emphasis should be placed on supporting information sharing, communication media, process management, privacy and flexible system configuration in order to facilitate the collaborative platform in digital environment. In addition, they emphasised that individual-oriented factors should also be considered in the development of a collaborative BIM platform.

In another study, Redmond et al. [6] focused on the importance of having an integrated platform for BIM applications (i.e. BIM Cloud) to enhance the BIM usability experience across the multi-organisations. They suggested that having such exchange mechanism will foster effective collaboration through sharing and exchanging data in order to provide more effective key decisions over time. Based on a case study of a large healthcare project (the project has been awarded BuildingSMART's 2015 award for 'outstanding open BIM practice') in Norway, Merschbrock and Munkvold [2] identified interorganizational factors driving the diffusion of BIM technology at the project level, and how individual, managerial, environmental, and technological challenges experienced by construction firms towards fostering a collaborative BIM workspace.

In contrast, Zheng et al. [4] focused on developing an outcome-linked benefit sharing model (OLBSM) to incentivize inter-firm BIM collaboration. They suggested that the successfulness of design and execution of an OLBSM depend on clear BIM objectives, procedural equities, and inclusive processes, as well as engage rigorous analysis of different incentive-alignment options to assess their possible effects on both beneficiaries and efforts. Poirier et al. [11] developed insights into the impact of BIM on collaboration in the architecture, engineering and construction industry in Canada. They identified five cognitive determinants to influence an individual's framing of BIM-enabled collaboration: requirements, expectations, intentions, incentives and capabilities.

Liu et al. [3], through a focus group discussion and interviews with BIM related participants, identified eight concepts (based on three dimensions; technology, people and process) influencing the development of BIM collaboration. On the other perspective, Kapogiannis, and Sherratt [12] focused on how integrated collaborative technologies have an impact on team collaboration between stakeholders. They found that collaborative culture could help provide access to information by stakeholders from anywhere at any time. In addition, in such a digital environment, stakeholders can share and access knowledge and awareness about the project and thus gain common ground and understanding about the project brief.

Overall, despite majority of these studies making significant progress in understanding the collaboration in BIM environment, they mainly attempt to discover the elements or factors influencing the BIM collaboration in different and several context rather than trying to consolidate what it takes to ensure the success of social collaboration in BIM. While collaboration in multiorganisations has proven to be challenging [8], social aspect of collaboration in BIM research has not received the level of attention it deserves [9]. Consequently, to promote understanding of the concept of social collaboration in BIM, this paper focuses on identifying the key elements of social collaboration and attempts to classify them, with the intention of gaining a greater insight into how they influence collaboration in BIMbased project.

\section{Research Methodology}

Initially in this review, papers relevant to team integration in construction projects published in the 
leading construction management journals and conferences were considered namely: ASCE Journal of Construction Engineering and Management (CEM), Automation in Construction (AIC), Construction Management and Economics (CME), Engineering, Construction, and Architectural Management (ECAM), International Journal of Project Management (IJPM), Journal of Information Technology in Construction, Construction Economics and Building, Built Environment Project and Asset Management and Association of Researchers in Construction Management.

The selection of these journals was based on their influence in the area of construction engineering and management based on their inclusion in the top-tier leading construction journals. Articles published from 2010 to 2018 were included in the literature search. The reason behind this is that the subject of collaboration in BIM emerged as a focus of research studies during this period as reported by Oraee et al. [9].

The initial article selections were conducted based on the guidelines made by several authors (e.g. Nakamba et al., [13]) in order to employ a systematic search to identify articles with the following phrases in subjects, titles, keywords or abstracts: BIM, social, collaboration, collaborative, construction. This search resulted in a broad selection of works that included empirical, qualitative, and descriptive articles, technical notes and book reviews. Consequently, to better reflect the scope of the study, a more focused search was carried out using two specific keywords within the initial BIM articles; collaboration and collaborative. In addition, the abstracts of all articles were briefly reviewed to determine their applicability. Finally, out of the 76 retrieved articles through initial selection, 27 articles related to BIM collaboration were reviewed.

\section{Nexus of Social Collaboration}

In this study, drawing upon the 27 research studies as well as four collaborative related models; Conceptual BIM Collaboration model developed by Lu et al. [14], Relationship-based Procurement (RBP) Collaboration Framework established by Walker and Lloyd-Walker [15], CoSpaces Collaborative Working Model (CCWM)) proposed by Patel et al. [16], and Collaboration Pentagon developed by Oraee et al. [9], the nexus of successful social collaboration have been synthesised into three main elements as illustrated in Fig. 1. These elements to social collaboration in BIM-based projects are Organisational context, Collaborative platform and Behavioural context. The following sections discuss each of these elements in turn.

\subsection{Foundational Platform}

Foundational Platform is seen as one of the most important elements that support the social collaboration in BIM. It is worth highlighting that BIM has been known as a platform of integration and management of coordination of multidisciplinary teams throughout the building life cycle $[3,5]$. The use of related social digital application in BIM-based project is beneficial as it could deliver precise construction informatics and providing greater visualisation, communication and interaction of via multi-dimensions modeling capabilities (e.g. social networking, message-based platform, cloud based information sharing). Several scholar (e.g. Liu et al. [3]; Singh et al. [10]) argued that greater emphasis should be placed on supporting technical requirements to facilitate technology application and management across the organisations for social interaction.

Apart from having collaborative digital technologies, other influencing mechanisms such contractual framework is vital to tackle and moderate more overarching variables (e.g. incentive, governance, legal, risks) that affect adoptability and use of BIM for social collaboration $[17,18]$. There is a need to move away from the fragmented practice that characterizes the project to an approach focused on more collaborative practice. Alternative contracting models such as integrated project delivery (IPD) and project alliance are proven to be successful platform for nurturing collaborative relationships through collaborative elements (e.g. incentive arrangements joint governance structure, co-location etc) $[15,19]$.

\subsection{Behavioural Context}

It is well acknowledged that behaviour is known to be the underlying and dominant factor in driving the social collaborative practice in digital environment [20]. Proactive behaviour is antecedent to embrace the collaborative culture (Kapogiannis and Sherratt, 2018). A combination of human-centric approach and behavioural best practices within the diverse team will ensure everyone is developing cross pollination of knowledge and skills towards common goal. Recognising the roles and responsibilities will embrace the collaboration that understand the synergistic aspects of the human relationships $[2,14]$.

In digital environment, actions of individuals may impact the collaboration due to changes of traditional roles [18]. Patel et al. [16] emphasised that organisations should be aware of the skills required to perform collaborative activities. Thus, embedding behavioural skills through digital literacy, knowledge and experience can drive the social normative practices towards collaboration movement. The integration of skills and expertise of individuals could enhance social networking [9].

\subsection{Organisational Context}

Collaboration in digital environment is mainly utilized by the multi-disciplinary organisations through its process [4, 14]. The significant impact on social collaboration within BIM would come from moving toward thinking about integrated organisation, where processes involved in delivering BIM-related task and activities are through collaborative working approaches. Consistency in interaction processes such as collective decision making, communication and information 


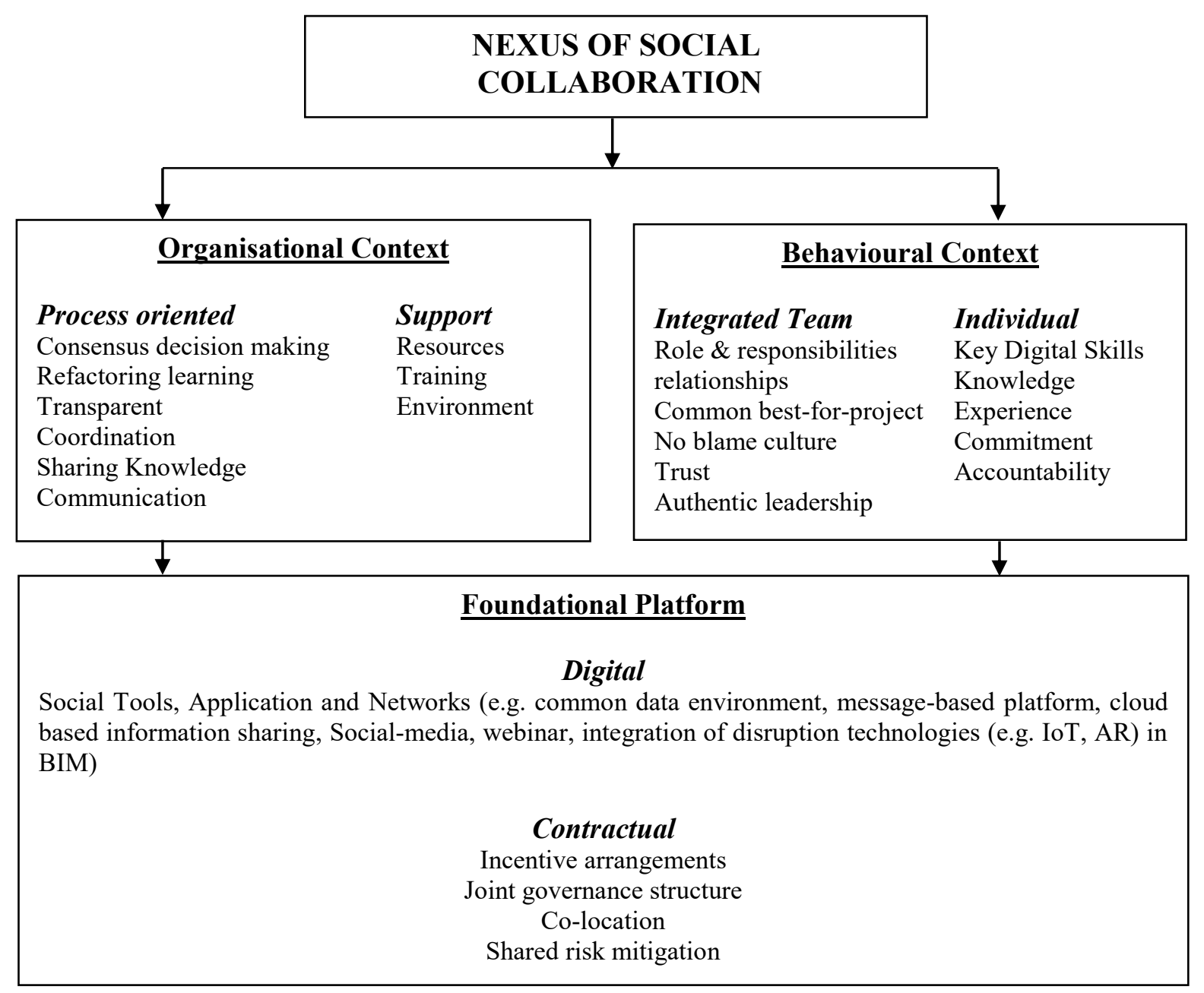

Fig. 1: Successful Social Collaboration Framework for BIM-based project

vital to increase the effectiveness of embracing the collaboration [16].

Another element that related under organisational context is the institutional support. The degree of institutional support that individuals receive can influence their commitment to the project and hence increase the fidelity of the project [14]. Kapogiannis and Sherratt [12] described that optimisation of organisational support in psychological perspectives could enhance team members' commitment in using collaborative technologies and hence improve their relationship and productivity. In addition, as highlighted by several scholars (e.g. [9,21]) is necessity of framing the BIM-enabled project within the collaborative environment (e.g. working and social space) to support the digital environment. Having such productive environment could influence positivity of social level between individual, discipline, team and organisation.

Overall, it can be seen that the need of social collaboration is significant in order to address the complex nature (e.g. socialisation, heterogeneous, and peculiar nature of projects) of the construction projects. BIM has evolves into becoming the central mean for integration and coordination of people, place, process and technology throughout a building's life cycle. Through the observation of the existing literature, effective social collaboration requires not only focusing beyond the technology-related features (which has been known as a central theme in BIM) but also to other elements notably behaviour and organisational related features.

However, it is worth highlighting that in all cases, the attempt is to influence the collaboration by having technological and contractual as a platform to govern and structure the pattern of the social collaboration. In addition, the processes involved, organisational support provided and instilling proactive and collective behaviour are also plays an important role to ensure the consistency of the sociotechnical aspect within the collaboration over time. Thus, the successful social collaboration in BIM-enabled project can only be achieved through purposeful integration of these three elements that fully support the end users' goals.

\section{Conclusion}

This paper presents a review of key elements of successful social collaboration in BIM-enabled project. In essence, the successfulness of social collaboration is a result of a combination of three key elements; Organisational context, Foundational platform and Behavioural context. The need of these three elements is

* Corresponding author: chekhairil449@salam.uitm.edu.my 
crucial to ensure the continuous interaction and positive relationships between the project team members.

It is worth highlighting that a widespread adoption of BIM in construction industry requires a highly social collaborative practice that can demonstrate the holistic approach in order to deliver full potential of BIM to the end user. Overall, the nexus of social collaboration (shown in Fig. 1) are considered as the theoretical lens of the future study. Consequently, the next phase of this research aims to expand this framework further, through validating and benchmarking to capture the gaps lie within these elements and their relationships within the digital context.

\section{References}

1. M. Mathews, D. Robles, B. Bowe, BIM+Blockchain : A Solution to the Trust Problem in Collaboration? CITA BIM Gathering 2017, November 23rd-24th November 2017 (2017)

2. C. Merschbrock, B.E. Munkvold, Effective digital collaboration in the construction industry - A case study of BIM deployment in a hospital construction project, Computers in Industry, 73, 1-7 (2015)

3. Y. Liu, S. van Nederveen, M. Hertogh, Understanding effects of BIM on collaborative design and construction : An empirical study in China, International Journal of Project Management, 35(4), 686-698 (2017)

4. L. Zheng, W. Lu, K. Chen, K.W. Chau, Y. Niu, Benefit sharing for BIM implementation: Tackling the moral hazard dilemma in inter-firm cooperation, International Journal of Project Management, 35(3), 393-405 (2017)

5. A.O. Akponeware, A. Zulfikar, Z.A. Adamu, Clash Detection or Clash Avoidance? An Investigation into Coordination Problems in 3D BIM, Buildings, 7(3), 1-28 (2017)

6. A. Redmond, A. Hore, M. Alshawi, R. West, Exploring how information exchanges can be enhanced through Cloud BIM, Automation in Construction, 24, 175-183 (2012)

7. T.D. Oesterreich, F. Teuteberg, Understanding the implications of digitisation and automation in the context of Industry 4.0: A triangulation approach and elements of a research agenda for the construction industry, Computers in Industry, 83, 121-139 (2016)

8. A. Kokkonen, V.L. Vaagaasar, Managing collaborative space in multi-partner projects. Construction Management and Economics, 36(2), 83-95 (2018)

9. M. Oraee, M. R. Hosseini, E. Papadonikolaki, R. Palliyaguru, M. Arashpour, Collaboration in BIMbased construction networks : A bibliometricqualitative literature review, International Journal of Project Management, 35, 1288 - 1301 (2017)

10. V. Singh N. Gu, X. Wang, A theoretical framework of a BIM-based multi-disciplinary collaboration platform, Automation in Construction, 20, 134-144 (2011)

11. E.A. Poirier, D. Forgues, S. Staub-French, Understanding the impact of BIM on collaboration : a Canadian case study, Building Research and Information, 45(6), 681-695 (2017)

12. G. Kapogiannis, F. Sherratt, Impact of integrated collaborative technologies to form a collaborative culture in construction projects, Built Environment Project and Asset Management, 8(1), 24-38 (2018)

13. C.C. Nakamba, P.W. Chan, M. Sharmina, How does social sustainability feature in studies of supply chain management? A review and research agenda, Supply Chain Management : An International Journal, 22(6), 522-541 (2017)

14. W. Lu, D. Zhang, S. Rowlinson, BIM collaboration : a conceptual model and its characteristics In: Smith, S.D and Ahiaga-Dagbui, D.D (Eds) Procs 29th Annual ARCOM Conference, 2-4 September 2013, Reading, UK, Association of Researchers in Construction Management, 25-34 (2013)

15. D.H.T. Walker, B.M. Lloyd-Walker, Collaborative Project Procurement Arrangements, Project Management Institute, Newtown Square, PA (2015)

16. H. Patel, M. Pettitt, J. R. Wilson, Factors of collaborative working : A framework for a collaboration model, Applied Ergonomics, 43, 1-26 (2012)

17. A.M. Mahamadu, L. Mahdjoubi, C.A., Booth, Challenges to digital collaborative exchange for sustainable project delivery through building information modeling technologies, WIT Transactions on Ecology and The Environment, 179(1), 547-557 (2013)

18. A. Porwal, K.N. Hewage, Building Information Modeling (BIM) partnering framework for public construction projects, Automation in Construction, 31, 204-214 (2013)

19. C.K.I. Che Ibrahim, S.B. Costello, S. Wilkinson, D. Walker, Innovation in alliancing for improved delivery of road infrastructure projects, International Journal of Managing Projects in Business, 10(4), 700-720 (2017)

20. N.H. Ibrahim, Reviewing the evidence : use of digital collaboration technologies in major building and infrastructure projects, Journal of Information Technology in Construction (ITcon), 18, 40-63 (2013)

21. E. Poirier, D. Forgues, S. Staub-french, Collaboration through innovation: implications for expertise in the AEC sector, Construction Management and Economics, 34, 769-789 (2016) 